\title{
LA RELACIÓN CLIMA-TURISMO: LA ISLA DE CALOR URBANO EN DOS CIUDADES TURÍSTICAS
}

\author{
Juan Antonio SERRA PARDO \\ NOTUS \\ juanantonioserrapardo@gmail.com
}

\section{RESUMEN}

El principal objetivo de la investigación es estudiar la isla de calor urbano (ICU) en dos ciudades mediterráneas (Ibiza y Lloret de Mar) cuya principal actividad es el turismo. Asimismo, también se observa si la actividad turística influye y/o modifica el fenómeno de la ICU. Ambas ciudades son importantes destinos turísticos que han experimentado procesos de rápida urbanización y dónde se producen tasas de ocupación hotelera mensual dispares, alcanzando los valores máximos en verano mientras que en invierno no se produce casi ocupación, dando lugar a dos periodos de actividad contrapuestos.

Mediante el método de los transectos o recorridos móviles se recogieron datos sobre el terreno en ambas ciudades durante cuatro campañas anuales, entre 2010 y 2014, divididas en dos periodos, estival e invernal, permitiendo generar una base de datos total de 22.760 valores térmicos, 12.400 en la ciudad de Ibiza y 11.360 en Lloret de Mar. El análisis estadístico permite caracterizar la ICU en ambas ciudades, mostrando la existencia de una ICU de intensidad moderada en ambas ciudades, cuya máxima intensidad absoluta se registra durante el periodo estival. Además, se incorpora la proposición de soluciones concretas para poder mitigar los efectos negativos de la ICU, en especial para el sector turístico. Respecto a la actividad turística y su influencia sobre la ICU, no se puede demostrar que ésta influya en la mayor o menor intensidad del fenómeno, aunque sí parece influir en la configuración espacial de la ICU en ambas ciudades.

Palabras clave: isla de calor urbano, mitigación, clima urbano, turismo, ciudades turísticas.

\section{ABSTRACT}

The main goal of the research is the study of the urban heat island (UHI) in two Mediterranean cities (Ibiza and Lloret de Mar), where the chief activity is tourism. Likewise, it observes if the tourist activity affects and/or changes the UHI. Both cities are important tourist destinations that have suffered a high-speed urban growth and show different monthly hotel occupancy rates. In summer, the rates reach maximum values while in winter the rates chop to zero. Therefore, two opposite periods exist with regard to the activity of the cities.

The method of mobile surveys was used to acquisition of data which allowed to gather data in both cities during four annual campaigns, between 2010 and 2014, carried out in summer and winter. This resulted in an important database with the 22.760 
thermal values, 12.400 in Ibiza and 11.360 in Lloret de Mar. The statistical analysis allows to characterise the UHI in both cities, showing the existence of a moderate UHI intensity in both, whose highest absolute intensity is in summer season. In addition, the study incorporates the proposal of solutions that might mitigate the negative effects of the UHIs, especially in the tourist sector. With regard to tourist activity and its influence on UHI, it has not been demonstrated that tourism has an influence on the higher or lower intensity of the phenomenon, even if it seems to influence on the UHI spatial configuration in both cities.

Key words: urban heat island, mitigation, urban climate, tourism, tourist cities.

\section{INTRODUCCIÓN}

La influencia del tiempo y el clima en la actividad humana, especialmente en las actividades económicas, ha sido percibida por el hombre desde la antigüedad y se ha presentado como un factor limitante o favorable para desarrollar ciertas actividades. En este caso, el ejemplo de la relación entre el clima y actividades económica que se quiere comentar es el binomio clima-turismo.

La Geografía se ha interesado por las relaciones existentes entre la distribución de la actividad humana y las variaciones espaciales del clima, por lo que también ha estudiado la relación entre los elementos climáticos y las actividades turísticas. Esta relación ha sido abordada desde la Geografía del Turismo, analizando principalmente el clima como soporte, factor de localización, recurso y atractivo turístico, y desde la Climatología en la creación de técnicas que permitan la evaluación del potencial turístico de los lugares (Gómez Martín, 1999).

Así varios estudios trataron el binomio clima-turismo a partir de que las condiciones climáticas, especialmente el clima local y la meteorología, condiciona la actividad turística (emplazamiento, calendario de actividad, tipología de productos, infraestructura, etc.). No obstante, el reconocimiento del cambio climático y de la influencia de la actividad antrópica en éste, genera la aparición de nuevas líneas de investigación. Por un lado, aparecen trabajos encaminados al estudio de medidas de adaptación a los impactos que tendrá el cambio climático sobre la actividad turística e introducirlas en la planificación. Por otro lado, se produce un replanteamiento de la relación clima-turismo y se pasa a considerar como el turismo, como actividad económica, influye sobre el clima. En este sentido, aparecen trabajos que analizan como la actividad turística contribuye al calentamiento global y medidas de mitigación pueden ser aplicadas en el sector.

Dentro de éstos últimos trabajos se puede situar parte de esta investigación. Se trata de un estudio de clima urbano, cuyo principal objetivo es estudiar la isla de calor urbano (ICU) en dos ciudades de rango medio cuya actividad económica más importante es el turismo y observar si esta actividad influye y/o modifica dicho fenómeno.

El término isla de calor urbana se utiliza para designar el fenómeno por el cual se observa el incremento de la temperatura del aire de las ciudades respecto a la de los alrededores. La ICU es uno de los mejores ejemplos de cómo la actividad humana impacta sobre el clima local. Además, se trata del elemento más significativo y reconocible del clima urbano, siendo el efecto mejor estudiado de la modificación climática 
provocada por la urbanización. Algunos investigadores reafirman a la ICU como uno de los principales problemas de este siglo derivado de la acción humana, especialmente en ciudades y áreas metropolitanas con grandes concentraciones de población y actividades económicas (Rizwan et al., 2008). El fenómeno de la ICU se asocia al deterioro de la calidad del aire urbano, el incremento del consumo energético, la mayor concentración de partículas contaminantes sobre áreas urbanas y el incremento de las tasas de mortalidad en las ciudades. Esto ha despertado un gran interés de científicos e ingenieros, lo que ha permitido establecer sus características, causas, impactos y consecuencias, en primer lugar, para luego desarrollar estrategias que permitan actuar sobre los impactos negativos ambientales y económicos que tiene sobre la sociedad y los beneficios asociados a medidas que ayudan a reducir o mitigar esos impactos.

Respecto a investigaciones que analicen la relación entre el turismo y la ICU, no son muy numerosas, como ejemplos pueden citarse una primera aproximación al estudio de la ICU en Ibiza (Serra Pardo, 2007) y un trabajo realizado en la ciudad china de Sanya, dónde se analiza el diferente comportamiento de la ICU antes, durante y después de las vacaciones del Año Nuevo Chino (Zhang y Wu, 2015). En el entorno mediterráneo, hay varios estudios en ciudades costeras que observan una mayor intensidad estival de la ICU (Founda et al., 2015), aunque parece atribuirse a la mayor radiación solar y al dominio de situaciones atmosféricas estables características de esa época y no al efecto del turismo (Vardoulakis et al., 2013). Otros trabajos se centran en analizar la ICU durante el verano en ciudades mediterráneas, ya que es cuando mayor incidencia tiene debido al incremento de disconfort térmico que conlleva (Giannaros y Melas, 2012), relacionado el fenómeno con episodios fuertes de calor (olas de calor), la calidad del aire (Poupkou et al., 2011) y la mayor demanda energética (Vardoulakis et al., 2013). Por último, se pueden mencionar algunas investigaciones que muestran cómo las actividades económicas influyen sobre la ICU, ya sea generando un diferente comportamiento espacio-temporal de la ICU (Fabrizi et al., 2010) o determinando que el máximo térmico se localice en áreas de importante actividad como zonas portuarias o áreas industriales (Stathopoulou y Cartalis, 2007).

\section{2. ÁREAS DE ESTUDIO}

La investigación se realiza en dos ciudades mediterráneas que representan importantes destinos turísticos, referentes a nivel internacional como son Ibiza y Lloret de Mar (fig 1). La ciudad de Ibiza, es la capital de la isla de Ibiza, cuenta con una población de 49.975 habitantes, una extensión del municipio de $11 \mathrm{~km}^{2}$ y una elevada densidad de 4.543,2 hab $/ \mathrm{km}^{2}$. Conviene señalar que el fenómeno urbano supera los límites municipales y se extiende por los municipios vecinos de Sant Josep y Santa Eulalia. La ciudad de Lloret de Mar, ubicada en el sector septentrional de la Costa Brava, tiene una población de 37.618 habitantes, una superficie de $48,7 \mathrm{~km}^{2}$ y una densidad $772,4 \mathrm{hab} / \mathrm{km}^{2}$.

Ambas son destinos de "sol y playa" mediterráneos que han experimentado procesos de rápida e incontrolada urbanización en el último cuarto del siglo XX, con fenómenos de especulación y fuerte impacto medioambiental sobre los ecosistemas y el paisaje, como el boom edilicio y de segundas casas, y donde se observan unas tasas de ocupación hotelera mensual muy dispar (tabla 1), alcanzando los valores máximos 
en los meses del verano mientras que en invierno no se produce ocupación, dando lugar a dos periodos de actividad contrapuestos. Esto significa una ciudad que presenta dos ritmos de vida urbana en un mismo espacio urbano, habiéndose de adaptar a una época de gran demanda de servicios y gran actividad humana, mientras que en otra época se caracteriza por una demanda de servicios más reducida y sectores urbanos que casi no registran actividad.

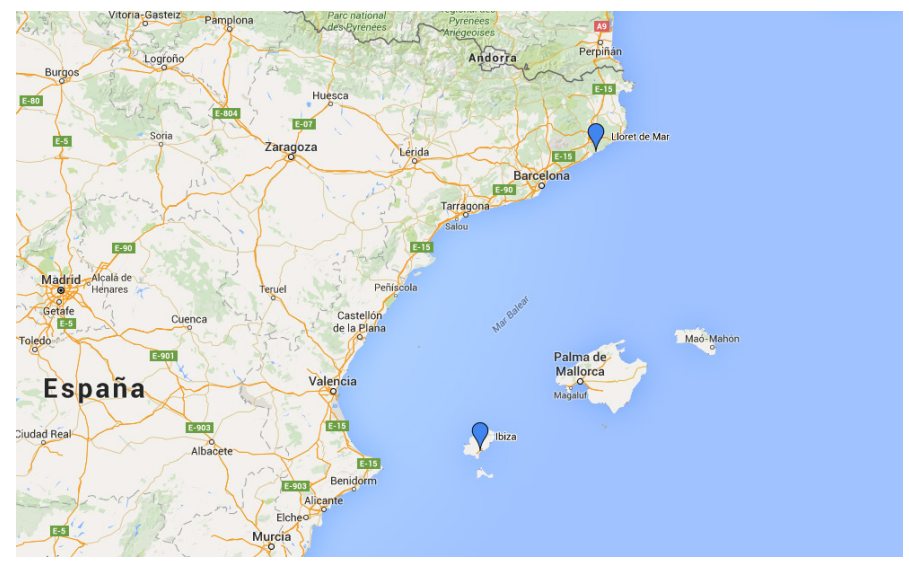

Fig. 1: Situación de Ibiza y Lloret de Mar. Fuente: Google Maps

\begin{tabular}{|l|c|c|c|c|c|c|c|c|c|c|c|c|}
\cline { 2 - 12 } \multicolumn{1}{c|}{} & E & F & Mr & A & My & Jn & Jl & A & S & O & N & D \\
\hline Ibiza &.. &.. & 28,9 & 56,2 & 63,5 & 82,1 & 93,3 & 95,5 & 85,2 & 55,6 &.. &.. \\
\hline $\begin{array}{l}\text { Lloret } \\
\text { de Mar }\end{array}$ &.. &.. & 61,9 & 62,2 & 51,0 & 69,5 & 77,3 & 89,0 & 74,3 & 68,9 & 49,1 & 41,6 \\
\hline
\end{tabular}

Tabla 1: Tasa de ocupación hotelera en 2014. Fuente: Encuesta de ocupación hotelera, INE

De este modo la actividad turística, en ambas ciudades, surge como principal agente transformador del territorio, factor de desarrollo de la actual morfología urbana, motor económico y dinamizador de sus economías locales y generador de importantes incrementos demográficos.

\section{METODOLOGÍA}

El método utilizado es la técnica de transectos o recorridos móviles. Dicho método consiste en la utilización de vehículos automóviles para realizar recorridos urbanos donde tomar medidas meteorológicas localizadas en diversos puntos a lo largo de los mismos, permitiendo obtener una serie de medidas meteorológicas sobre el terreno. De esta manera, el método de los transectos pone el acento, mediante el trabajo de campo, en la obtención de una serie de medidas que van a incidir más sobre la distribución espacial y la configuración del fenómeno de la ICU.

En la ciudad de Ibiza se diseñan un total de tres transectos que suman una longitud de $26,3 \mathrm{~km}$ y se establecen un total de 67 puntos de observación donde realizar la 
toma de temperaturas. En el caso de Lloret de Mar se diseñan también tres transectos que presentan una longitud total de $18,9 \mathrm{~km}$ y 47 puntos de observación distribuidos por la ciudad. Los transectos presentan puntos en común entre sí para permitir el ajuste en las temperaturas anotadas y, así, poder ser consideradas como simultáneas.

Los datos se registran mediante dos campañas anuales, una, en invierno, durante los meses de diciembre a marzo, y otra, en verano, durante los meses de junio a septiembre, que se desarrollarán durante 4 años. Entre el 1 de julio de 2010 y el 4 de abril de 2014, durante 315 noches, se recopilan un total de 22.760 valores térmicos, de los que 10.360 son en Lloret de Mar y 12.400 en Ibiza.

\section{RESULTADOS}

El análisis estadístico de la amplia base de datos permite caracterizar la ICU (intensidad, configuración espacial y localización del máximo) en ambas ciudades. Además, se establecen correlaciones entre la intensidad de la ICU y otros parámetros como: la presión atmosférica, la situación sinóptica y el día de la semana para ambas ciudades y, en el caso de Ibiza, la velocidad del viento, visibilidad y energía eléctrica. Además del análisis general de los datos, también se realiza la comparación entre los periodos estacionales (invierno y verano) para observar si influye o no la actividad turística sobre la isla de calor $y$, por tanto, sobre el clima urbano.

\subsection{Ibiza}

Los resultados muestran la existencia de una ICU moderada en esta ciudad, con una intensidad media de $3,6^{\circ} \mathrm{C}$. La intensidad media invernal $\left(3,8^{\mathrm{a}} \mathrm{C}\right)$ en la ciudad es $0,3^{\circ} \mathrm{C}$ mayor que la estival $\left(3,5^{\circ} \mathrm{C}\right)$. La máxima intensidad absoluta se registra durante el periodo estival, el 5 de agosto de 2010 se observa una intensidad de $7,5^{\circ} \mathrm{C}$, siendo $0,5^{\circ} \mathrm{C}$ superior a la máxima intensidad invernal, registrada 25 de diciembre de 2010 con $7^{\circ} \mathrm{C}$.

En la tabla 2 se muestran las intensidades de la ICU de Ibiza, clasificadas según la propuesta de Fernández (1996). A nivel general, se contempla que las intensidades moderadas (de $2^{\circ} \mathrm{C} \mathrm{a} 4^{\circ} \mathrm{C}$ ) son las que más frecuentemente se han registrado durante el análisis de la ICU de Ibiza. Aunque también resulta destacable el alto número de días con una intensidad fuerte $\left(\operatorname{de~} 4^{\circ} \mathrm{C}\right.$ a $\left.6^{\circ} \mathrm{C}\right)$. Analizando los datos según en la época donde se obtienen las intensidades, destaca cómo en invierno se produce una mayor heterogeneidad, con presencia de intensidades débiles $\left(\right.$ de $\left.0^{\circ} \mathrm{C} \mathrm{a} 2^{\circ} \mathrm{C}\right)$ y con intensidades muy fuertes (de $6^{\circ} \mathrm{C}$ o más). Mientras que durante el verano se contemplan unas intensidades más homogéneas, donde casi $2 / 3$ de los días presentan una intensidad moderada y muy pocas veces se producen intensidades débiles o muy intensas.

\begin{tabular}{|lrrrrrr|}
\hline Intensidad & \multicolumn{2}{c}{ Verano } & \multicolumn{2}{c|}{ Invierno } & \multicolumn{2}{c|}{ Total } \\
Débil & 5 & $6,7 \%$ & 15 & $18,8 \%$ & 20 & $12,9 \%$ \\
Moderada & 46 & $61,3 \%$ & 24 & $30,0 \%$ & 70 & $45,2 \%$ \\
Fuerte & 22 & $29,3 \%$ & 29 & $36,3 \%$ & 51 & $32,9 \%$ \\
Muy fuerte & 2 & $2,7 \%$ & 12 & $15,0 \%$ & 14 & $9,0 \%$ \\
\hline Totales & 75 & $100,0 \%$ & 80 & $100,0 \%$ & 155 & $100,0 \%$ \\
\hline
\end{tabular}

Tabla 2: Clasificación de las intensidades de la ICU en Ibiza 
La ICU muestra una configuración espacial que suele ser de forma concéntrica sobre el área central de la ciudad (Eixample). Resulta destacable cómo durante las diferentes campañas se advierte que la extensión del núcleo de la ICU varía. En general, durante el verano se observa un núcleo menos extenso, mientras que en invierno el centro térmico presenta una mayor extensión. Otro aspecto reseñable es el diferente comportamiento térmico que manifiestan los sectores turísticos de la ciudad, en especial Platja d'en Bossa, según la época del año. Así, como muestra la fig 2., en las campañas estivales estas áreas tienen un comportamiento térmico más cálido, similar al del área central de la ciudad, mientras que en invierno presentan una temperatura más fresca, especialmente bajo la influencia de los humedales próximos (Prat d'en Fita y Ses Feixes).

a)

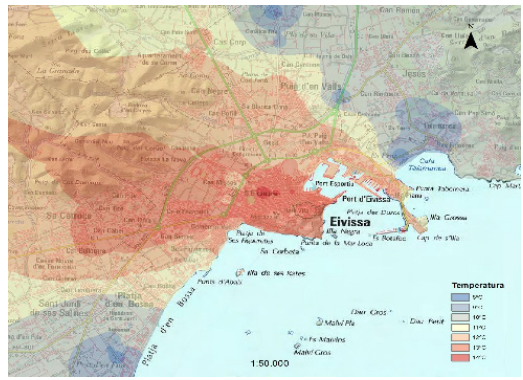

b)

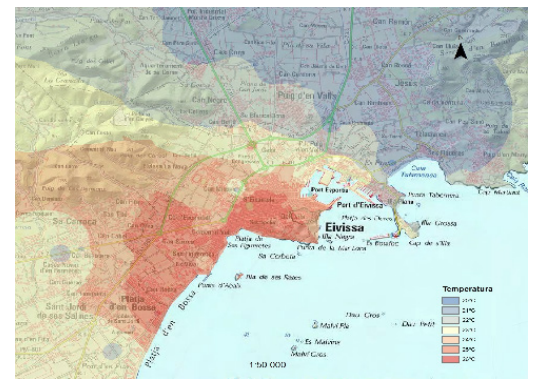

Fig. 2: Configuración espacial de la ICU en Ibiza a) Día 9 de agosto de 2010, b) Día 23 de diciembre de 2013. Fuente: Elaboración propia a partir de la Base cartográfica del IGN

Respecto a las correlaciones establecidas entre la intensidad de la ICU y otros parámetros, se ha observado que la presencia de situaciones atmosféricas estables y dominadas por altas presiones favorecen unas mayores intensidades, así los días con situaciones anticiclónicas presentan una intensidad media de $3,8^{\circ} \mathrm{C}$. En sentido contrario, la velocidad del viento aparece como un factor que debilita la intensidad de la ICU, presentando una correlación negativa de 0,389. Al analizar las intensidades medias según el día de la semana, parece que los fines de semana hay unas mayores intensidades de la ICU. Aunque el parámetro que ha obtenido una mayor correlación, del 0,644, ha sido el retraso de la hora en que se produce el pico de demanda eléctrica y la mayor intensidad.

\subsection{Lloret de Mar}

En esta ciudad gerundense se observa una ICU moderada, cuya intensidad media es de $3,3^{\circ} \mathrm{C}$. La intensidad media estival $\left(3,5^{\circ} \mathrm{C}\right)$ es $0,4^{\circ} \mathrm{C}$ superior a la invernal $\left(3,1^{\circ} \mathrm{C}\right)$. La intensidad máxima se obtiene en verano, el 10 de julio de 2010 se registra $6,6^{\circ} \mathrm{C}$, y es $1,1^{\circ} \mathrm{C}$ mayor a la máxima intensidad invernal, recogida el 28 de febrero de 2013 con $5,5^{\circ} \mathrm{C}$ su configuración espacial muestra una forma semicircular con el núcleo sobre el frente litoral del centro histórico de la ciudad.

La clasificación de las intensidades de la ICU (tabla 3) permite observar cómo las intensidades moderadas se dan con mayor frecuencia, en algo más de la mitad de los días analizados. También resultan destacables las jornadas que manifiestan una 
intensidad fuerte, representando casi $1 / 3$ del total. Por último, hay que señalar que en invierno es cuando se da un mayor número de días con una ICU débil.

\begin{tabular}{|lrrrrrr|}
\hline Intensidad & \multicolumn{2}{c}{ Verano } & \multicolumn{2}{c}{ Invierno } & \multicolumn{2}{c|}{ Total } \\
\hline Débil & 5 & $6,3 \%$ & 15 & $18,8 \%$ & 20 & $12,5 \%$ \\
Moderada & 50 & $61,3 \%$ & 40 & $50,0 \%$ & 89 & $55,6 \%$ \\
Fuerte & 25 & $31,3 \%$ & 25 & $31,3 \%$ & 50 & $31,3 \%$ \\
Muy fuerte & 1 & $1,3 \%$ & 0 & $0,0 \%$ & 1 & $0,6 \%$ \\
\hline Totales & 80 & $100 \%$ & 80 & $100,0 \%$ & 160 & $100,0 \%$ \\
\hline
\end{tabular}

Tabla 3: Clasificación de las intensidades de la ICU en Lloret de Mar

a)

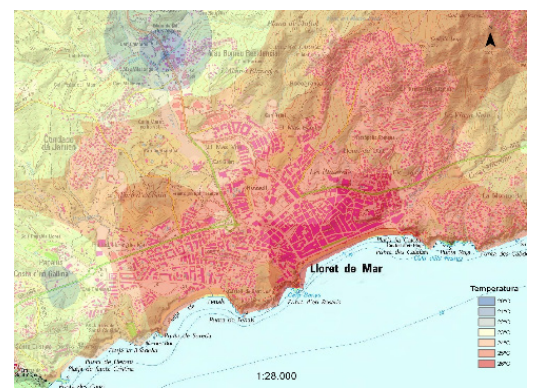

b)

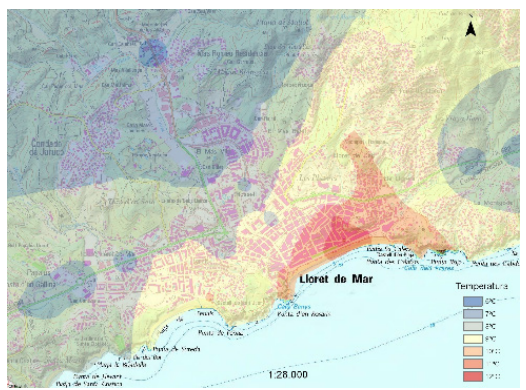

Fig. 3: Configuración espacial de la ICU en Lloret de Mar a) Día 17 de julio de 2011, b) Día 9 de febrero de 2011. Fuente: Elaboración propia a partir de la Base cartográfica del IGN

La configuración espacial de la ICU de Lloret de Mar muestra una forma semicircular con su máximo térmico ubicado sobre el frente litoral del centro histórico de la ciudad. Cabe destacar cómo la extensión del máximo térmico ocupa una mayor o menor extensión en las distintas campañas. Por lo general, en verano surge un centro térmico más extenso que penetra hacia el interior del núcleo antiguo de la ciudad y hacia el sector turístico colindante, mientras que en invierno el centro térmico ofrece una menor extensión, ubicándose de forma más localizada en el frente litoral (véase fig. 3). La actividad turística, gran parte localizada en el barrio del Nucli Antic, parece afectar que el centro térmico extienda su influencia sobre una mayor superficie de la ciudad.

En el caso de Lloret de Mar, las correlaciones analizadas señalan cómo las intensidades máximas se ven favorecidas por la presencia de altas presiones, de este modo las situaciones anticiclónicas muestran una intensidad media de $3,9^{\circ} \mathrm{C}$. El análisis de las intensidades medias según el día de la semana no permite establecer tendencias claras, no obstante parece que los sábados presentan una mayor intensidad.

\section{DISCUSION}

La investigación no permite demostrar que la actividad turística influya en la mayor o menor intensidad de la ICU en Lloret de Mar y en Ibiza y sí que parece influir en la configuración espacial del fenómeno en ambas ciudades. 
Los datos examinados resultan insuficientes para poder analizar qué grado de importancia tiene la actividad turística en que durante la época estival se haya alcanzado una máxima intensidad.

En cambio, en la configuración espacial de la ICU se evidencian diferencias estacionales que sí pueden ser atribuibles a la actividad turística. En especial existen dos aspectos, el primero es la diferente extensión y localización del máximo térmico de la ICU en ambas ciudades. En el caso de Lloret de Mar, en verano el máximo térmico presenta una mayor superficie al extenderse por sectores urbanos donde se intensifican la actividad durante la temporada turística, mientras que en invierno el máximo térmico tiene una menor superficie y se encuentra más localizado. En el caso de Ibiza, el máximo térmico presenta una diferente localización. El segundo aspecto, es la existencia de sectores urbanos, donde domina o tiene una importante presencia la función turística, que presentan un diferente comportamiento térmico estacional atribuible a la apertura de establecimientos hoteleros y locales relacionados con actividades turísticas durante la temporada. Estos sectores tienen un comportamiento más cálido y cercano a los centros térmicos de la ICU durante el verano, pudiendo localizarse núcleos secundarios o incluso, en alguna ocasión, el núcleo principal de la ICU. Un ejemplo sería Platja d'en Bossa y Figueretas en Ibiza, mientras que en Lloret de Mar los ejemplos estarían en Fenals y el barrio de la Riera.

Además del análisis estadístico, la investigación incorpora una vertiente aplicada mediante la proposición soluciones concretas para poder mitigar los efectos negativos del fenómeno de la ICU, especialmente en el sector turístico. En este sector se pueden aplicar medidas destinadas a mitigar sus efectos pensando en mejorar, en general, la calidad del aire, y, en especial, la eficiencia energética a partir de disminuir la mayor demanda estival de energía, especialmente la destinada a mejorar el confort térmico de los turistas (aires acondicionados). Por ejemplo, en el caso de Ibiza la demanda de energía durante el verano se incrementa un promedio del $85 \%$ respecto a la invernal. Así que, el principal problema en estas dos ciudades sería el incremento del disconfort térmico que supone la ICU, sobre todo nocturno, que genera un mayor consumo energético y la introducción de medidas de eficiencia energética que reduzcan ese consumo.

Los hoteles representan una porción significativa de las edificaciones en ambas ciudades, constituyendo una parte fundamental del incremento de la demanda de energía estival, por lo que aplicar medidas de eficiencia energética sobre la infraestructura hotelera se traducirá en importantes beneficios. Las medidas pueden ser de carácter opcional que puedan efectuar los hoteleros por voluntad propia o por la existencia de incentivos o bien de carácter obligatorio, mediante la elaboración de una legislación por parte de la administración pública. Las medidas a aplicar podrían ser:

a) La utilización de materiales fríos. El uso de pinturas con un alto albedo es una medida fácil de aplicar y económica. Un ejemplo sería los techos fríos mediante el pintado de blanco. Antes de iniciar la temporada, los hoteles deben acometer una puesta a punto u obras de mejora, momento apropiado para aplicar esta medida.

b) Instalación de paneles solares y termo solares en los techos de los hoteles. Por un lado, los paneles solares suponen un beneficio económico ya que una parte de la demanda energética queda satisfecha por el autoconsumo, y por otro, estos reducen la radiación solar absorbida por el propio edificio que se traduciría en una menor necesidad de refrigeración para lograr un ambiente agradable. 
c) Fomentar el uso de aparatos eléctricos de alta eficiencia energética e iluminación de baja intensidad. Antes del inicio de cada temporada debería ser necesario presentar una evaluación de la eficiencia energética del establecimiento, incluyendo mejoras realizadas respecto al año anterior.

d) Incrementar las superficies verdes alrededor de los edificios hoteleros. En los hoteles de nueva construcción, se podría obligar mediante ordenanzas. Otro modo de incrementar las superficies verdes en los hoteles se puede ser mediante la introducción de vegetación alrededor de los espacios exteriores, como terrazas o piscinas, o creando espacios verdes para el uso de clientes en las azoteas o generando muros verdes. Esto también se traduciría en una mejor estética de los establecimientos por suponer un elemento ornamental.

e) Asegurar un buen aislamiento térmico. La revisión periódica, quizás en el comienzo de cada campaña, que no haya perdidas de aislamiento debido a puertas o ventanas que no cierran bien. También se deben contemplar la instalación de cierres automáticos, ya sea electrónico o mecánico, en puertas que den a espacios exteriores.

f) Elaborar y distribuir trípticos sobre criterios de sostenibilidad y eficiencia energética para que los clientes tengan en cuenta durante su estancia, como por informar que no tengan el aire acondicionado encendido con la puerta del balcón abierto.

g) Fomentar entre los establecimientos la incorporación de criterios de eficiencia para obtener certificados internacionales que supongan un sello de calidad que despierte interés entre posibles clientes concienciados ambientalmente.

\section{REFERENCIAS}

Fabrizi, R., Bonafoni, S. y Biondi, R. (2010). Satellite and Ground-Based Sensors for the Urban Heat Island Analysis in the City of Rome. Remote sensing, vol. 2, $\mathrm{n}^{\circ} 5$, pp. 1400-1415. Doi: http://dx.doi.org/10.3390/rs2051400

Founda, D., Pierros, F., Petrakis, M. y Zerefos, C. (2015). Interdecadal variations and trends of the Urban Heat Island in Athens (Greece) and its response to heat waves. Athmospheric Research, vol. 161-162, pp. 1-13. Doi: http://dx.doi.org/10.1016/j. atmosres.2015.03.016

Fernández García, F. (1996). Manual de Climatología Aplicada: clima, medio ambiente y planificación. Madrid: Síntesis.

Giannaros, T.M. y Melas, D. (2012). Study of the urban heat island in a coastal Mediterranean City: The case study of Thessaloniki, Greece. Atmosphere Research, vol. 118, pp. 103-120. Doi: http://dx.doi.org/10.1016/j.atmosres.2012.06.006

Gómez Martín, M. B. (1999). La relación clima-turismo: consideraciones básicas en los fundamentos teóricos y prácticos. Investigaciones Geográficas, nº 21, pp. 21 34. Doi: http://dx.doi.org/10.14198/ingeo1999.21.04

Poupkou, A., Nastos, P., Melas, D. y Zerefos, C. (2011). Climatology of Discomfort Index and Air Quality Index in a Large Urban Mediterranean Agglomeration. Water Air Soil \& Polluntion, vol. 222, no 1, pp. 163-183. Doi: http://dx.doi. org/10.1007/s11270-011-0814-9 
Rizwan, A. M., Dennis, L. y Liu, C. (2008). A review on the generation, determination and mitigation of urban heat island. Journal of Environmental Sciences, vol. 20, $\mathrm{n}^{\circ}$ 1, pp. 120-128. Doi: http://dx.doi.org/10.1016/S1001-0742(08)60019-4

Serra Pardo, J.A. (2007). Estudio de la isla de calor de la ciudad de Ibiza. Investigaciones Geográficas, $\mathrm{n}^{\circ}$ 44, pp. 55-73. Doi: http://dx.doi.org/10.14198/ingeo2007.44.03

Stathopoulou, M. y Cartalis, C. (2007): Daytime urban heat islands from Landsat ETM+ and Corine land cover data: An application to major cities in Greece. Solar Energy, vol. 81, $\mathrm{n}^{\mathrm{o}}$ 3, pp. 358-368. Doi: http://dx.doi.org/10.1016/j. solener.2006.06.014

Vardoulakis, E., Karamanis, D., Fotiadi, A. y Mihalakakou G. (2013). The urban heat island effect in a small Mediterranean city of high summer temperatures and cooling energy demands. Solar Energy, vol. 94, pp. 128-144. Doi: http://dx.doi. org/10.1016/j.solener.2013.04.016

Zhang, J. y Wu, L. (2015). Modulation of the urban heat island by tourism during the Chinese New Year holiday: a case study in Sanya City, Hainan Province of China. Science Bulletin, vol. 60, $\mathrm{n}^{\circ}$ 17, pp. 1543-1546. Doi: http://doi.org/10.1007/ s11434-015-0864-2 